\title{
Acute visual loss without concurrent headaches due to ultrasound-negative, biopsy-proven giant cell arteritis
}

\author{
Authors: Ruchir Singh, ${ }^{\mathrm{A}}$ Tharshini Rajakulenthiran, ${ }^{\mathrm{A}}$ Sethuge Silva ${ }^{\mathrm{A}}$ and Roshan Amarasena ${ }^{\mathrm{A}}$
}

\section{Introduction}

Giant cell arteritis (GCA) is a vasculitis caused by immune cascades resulting in vascular inflammation, remodelling and vessel occlusion, and therefore a cause of arteritic anterior ischaemic optic neuropathy. ${ }^{1}$ Characteristically, it is accompanied by headaches, jaw claudication, scalp tenderness and constitutional symptoms. This case represents an atypical presentation of GCA, with sudden vision loss in the absence of concurrent characteristic symptoms.

\section{Materials and methods}

An 82-year-old Caucasian woman presented with blurring and greying of her peripheral visual fields in the right eye. She later returned home due to extended wait times in emergency department, and opted to return in the morning. Overnight her symptoms progressed to acute total visual loss and she re-presented. On admission she had no other symptoms and was thus reviewed by ophthalmology. She had right optic disc swelling and vision limited to hand movements only. On further history, she mentioned a 6-week history of temporal tenderness, pain on brushing hair, polymyalgia and weight loss. Interestingly, these symptoms had resolved 2 weeks prior spontaneously, and she had not sought medical help for these as she was unaware of their importance. On repeat examination, she was noted to have reduced right temporal artery pulsation. Despite the atypical presentation, and given the acute vision loss, she was treated with a 3 -day course of pulsed intravenous methylprednisolone.

\section{Results and discussion}

Inflammatory markers were raised (C-reactive protein (CRP) $44 \mathrm{mg} / \mathrm{L}$, erythrocyte sedimentation rate (ESR) $81 \mathrm{~mm} / \mathrm{hr}$ ). Computed tomography (CT) of the head showed no ischaemia or haemorrhages. An ultrasound doppler of the temporal arteries revealed reduced size and flow of the right temporal artery, but no inflammation or focal arterial wall thickening. However, this was performed while on immunosuppression. Given the sight loss, a temporal artery biopsy (TAB) was

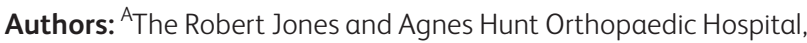
Oswestry, UK undertaken. This showed active, chronic transmural inflammation and periarterial fibrosis with giant cells within the internal elastic lamina. This confirmed GCA and the patient was switched to oral glucocorticoids. Her inflammatory markers reduced (CRP $6 \mathrm{mg} / \mathrm{L}$, ESR $28 \mathrm{~mm} / \mathrm{hr}$ ) and at 4-week follow up there was no relapse or contralateral vision loss.

\section{Conclusion}

This case provides several learning points for general physicians. GCA is a common life-changing disease seen on the acute take, resulting in irreversible visual loss. 1) Visual complications of GCA merits high-dose glucocorticoid therapy, as do features predicative of visual loss such as diplopia, jaw claudication and temporal artery abnormalities. 2) Resolution/ absence of typical symptoms do not rule out disease activity, nor the risk of visual sequelae, as this patient developed rapid vision loss despite the absence of concurrent temporal headache, polymyalgia or scalp tenderness. 3) It is paramount to enquire regarding the characteristic symptoms of GCA, both past and present, in patients presenting with sudden vision loss. 4) Although temporal artery ultrasound provides a quick, noninvasive method for identification of GCA whilst an inpatient, TAB can remain positive for up to 6 weeks following the initiation of immunosuppressant therapy. ${ }^{2}$ ) Performing TAB in those with a negative ultrasound increases the sensitivity and specificity of GCA diagnosis, ${ }^{3}$ and thus should be arranged for prior to discharge.

\section{Conflicts of interest}

None declared.

\section{References}

1 UpToDate. Pathogenesis of giant cell arteritis. UpToDate, 2019. www.uptodate.com/contents/pathogenesis-of-giant-cell-arteritis/ abstract/1,2 [Accessed 20 October 2019].

2 Dasgupta B, Borg FA, Hassan N et al. BSR and BHPR guidelines for the management of giant cell arteritis. Rheumatology (Oxford) 2010;49:1594-7.

3 Luqmani R, Lee E, Singh S et al. The role of ultrasound compared to biopsy of temporal arteries in the diagnosis and treatment of giant cell arteritis (TABUL): a diagnostic accuracy and costeffectiveness study. Health Technol Assess 2016;20:1-238. 